\title{
Selection of large quantities of embryogenic calli from indica rice seeds for production of fertile transgenic plants using the biolistic method
}

\author{
Elumalai Sivamani, Ping Shen, Natacha Opalka, Roger N. Beachy, and Claude M. Fauquet \\ ILTAB/TSRI-ORSTOM, The Scripps Research Institute, Division of Plant Biology, \\ 10666 North Torrey Pines Road-MRC7, La Jolla, CA 92037, USA
}

Received 27 February 1995/Revised version received 29 June 1995 - Communicated by I. K. Vasil

\begin{abstract}
The microprojectile bombardment of immature embryos has proven to be effective in transforming many indica rice varieties. One of the drawbacks of using immature embryos is the requirement of a large number of high quality immature embryos, which itself is a tedious and laborious process. To circumvent these problems, we have developed a procedure, using indica variety TN1 as a model that generates highly homogenous populations of embryogenic subcultured calli by selectively propagating a small number of regeneration-proficient calli derived from seeds. Thousands of embryogenic calli were produced from 50 seeds within 10 weeks. Ten to 20 independent RO transgenic lines were regenerated per 500 embryogenic calli bombarded. The convenience and reliability offered by this transformation system has made transformation of indica rice a routine procedure.
\end{abstract}

Abbreviation: 2,4-D: 2,4-dichlorophenoxy acetic acid; NAA: naphthalene acetic acid; BAP: 6-benzylaminopurine; kb: kilobase; GUS: $\beta$-glucuronidase; X-gluc: 5-bromo-4chloro-3-indolyl- $\beta$-D-glucuronide; HPH: hygromycin $B$ phosphotransferase

\section{Introduction}

Genetic engineering is considered to be an efficient approach for improving and creating agronomically valuable traits of crop plants. In the past decade, extensive research efforts have been focused on rice (Oryza sativa $\mathrm{L}$ ) with the aim of establishing an efficient and reliable rice transformation system. To date, two transformation techniques, protoplast transformation mediated by polyethylene glycol or by electroporation, and microprojectile bombardment of immature embryos or embryogenic calli, have been used routinely to transform the japonica subspecies of rice (Toriyama et al., 1988; Zhang et al., 1988; Zhang and Wu, 1988; Shimamoto et al., 1989; Datta et al., 1990; Peng et al., 1992; Li et al., 1993). While fertile transgenic plants can be obtained using either method, the biolistic method is becoming the method of choice because it alleviates the need of preparing protoplasts, reduces the time needed to regenerate transgenic plants, and results in transgenic plants with higher fertility.
Most recently, Agrobacterium-mediated transformation of japonica rice has been developed, and provides another alternative technique to protoplast transformation (Chan et al., 1993; Hiei et al., 1994).

In contrast to japonica rice, the indica subspecies of rice, which is economically more important, is generally more difficult to transform due to lower regenerability. Although a number of indica rice transformation systems have been reported (Datta et al., 1990; Peng et al., 1992; Christou et al., 1991; Xu and Li, 1994; Ghosh Biswas et al., 1994), most of them have not gained popularity due to problems of poor reproducibility, low fertility and/or technical complexity of the method. The microprojectile-mediated transformation system developed by Christou et al. (1991) has good reproducibility and is technically simple. In this system, immature embryos are used as target materials, and 1-2 transgenic plant lines can generally be regenerated from 100 bombarded immature embryos. However, this method also suffers from several drawbacks. First, to obtain a few fertile transgenic lines that exhibit proper expression of the gene of interest, it is necessary to prepare hundreds and, sometimes, thousands of immature embryos, which in itself is a tedious and labor intensive process. Second, a high quality greenhouse is required to maintain a constant source of high quality immature embryos. Furthermore, the quality and the yield of immature embryos are very poor in winter months in many geographic locations.

These problems prompted us to search for alternative sources of target materials for microprojectile bombardment. To this end, the indica rice variety TN1, was chosen as a model for our study. TN1 is an important breeding line used at the International Rice Research Institute, The Philippines. It is also a host of choice for testing genetically engineered disease resistance due to its high susceptibility to many rice diseases. To date, transformation of TN1 using microprojectile bombardment of immature embryos has been rather inefficient (unpublished results). In this paper, we report the successful development of a procedure for selectively propagating large quantities of highly embryogenic calli derived from mature TN1 seeds. We further demonstrate that these calli are suitable target materials for microprojectile bombardment. The 
convenience and reliability offered by this transformation system has made the production of transgenic indica rice plants a routine practice in this laboratory.

\section{Materials and Methods}

Seeds TN1 rice seeds were provided by the International Rice Research Institute (IRRI, Los Baños, The Philippines). TNI rice is considered to be a "class I" indica variety according to Glaszman (1987).

Media NB medium is composed of N6 macro elements (Chu et al., 1975), B5 micro elements and vitamins (Gamborg et al., 1968), $300 \mathrm{mg} / \mathrm{l}$ casein hydrolysate, $500 \mathrm{mg} / \mathrm{L}$-proline, $30 \mathrm{~g} / 1$ sucrose, $2 \mathrm{mg} / \mathrm{l} 2,4-\mathrm{D}$, and $0.4 \%$ agarose (type I, low EEO; Sigma, St. Louis, MO). The NBKNB medium is NB medium supplemented with $1 \mathrm{mg} / \mathrm{l}$ kinetin; $1 \mathrm{mg} / \mathrm{NAA}$ and $1 \mathrm{mg} / 1 \mathrm{BAP}$. RN medium is the same as NBKNB medium except with $0.5 \mathrm{mg} / 1 \mathrm{NAA}, 3 \mathrm{mg} / 1 \mathrm{BAP}$ and without $2,4-\mathrm{D}$. Rooting medium is half-strength MS medium (Murashige and Skoog, 1962) supplemented with $1 \mathrm{mg} / \mathrm{N}$ NAA and $1 \mathrm{mg} / \mathrm{l}$ BAP. The $\mathrm{pH}$ of media was adjusted to 5.8 with $1 \mathrm{M} \mathrm{KOH}$, prior to being autoclaved at $121^{\circ} \mathrm{C}$ for $20 \mathrm{~min}$.

Plasmids The plasmid, pILTAB222, was used for transformation experiments. This plasmid was constructed by inserting the hygromycin B phosphotransferase coding sequence into the BamHI site of pAHC17 (kindly provided by P. Quail). pAHC17 contains the maize ubiquitin (UBI) promoter, the first UBI intron and the nos 3 ' terminator (Christensen et al, 1992). Plasmid pAHC27, also provided by $P$. Quail, is a derivative of pAHC17 containing a GUS gene under the control of the maize UBI promoter. The plasmid DNA used for microprojectile bombardment was purified using the purification columns purchased from Qiagen Inc. (Chatsworth, CA).

Callus induction and selection of regenerable calli The procedure for generating and maintaining highly regenerable embryogenic callus is schematically summarized in Figure 1. Dehusked mature rice seeds were surface-sterilized in $70 \%$ ethanol for $2 \mathrm{~min}$. followed by $25 \%$ commercial bleach ( $1.3 \%$ sodium hypochlorite) with $0.02 \%$ Tween 20 for $45 \mathrm{~min}$. The seeds were then rinsed 5 times with copious amounts of sterilized water. The seeds were placed on petri dishes containing NBKNB medium, and incubated at $25^{\circ} \mathrm{C}$ for 6 days in the dark. At this point, the emerging primary calli induced from the scutellar region were removed from the rest of seeds, and subcultured in fresh NBKNB medium at $25^{\circ} \mathrm{C}$ for 2 weeks in the dark. Two more tounds of subcultured were carried out in the same manner. Before each subculture, the large primary calli were cut so that their initial were maintained at around $3-5 \mathrm{~mm}$ in diameter. After three 2-week subculture cycles, many loosely attached small globular calli appeared on top of each compact primary callus, which were gently removed with a pair of forceps. The small calli of $1-3 \mathrm{~mm}$ in diameter were individually transferred to plates containing fresh medium marked with numbered grids. After 2-weeks, a cluster of small globular calli had emerged on most calli, and a representative piece from each grid was transferred to a corresponding grid in plates containing $\mathrm{RN}$ medium. These calli were tested for regeneration for 10 days in a growth room (constant temperature of $30^{\circ} \mathrm{C} ; 16 \mathrm{~h}$ day $/ 8 \mathrm{~h}$ night; fluores- cent illumination of $100-125 \mathrm{~mm} / \mathrm{m}^{2} / \mathrm{s}$ PAR). All the calli that produced small shoots (Fig. 3 ) within 10 days were marked, and their sibling calli were collected for biolistic transformation or further multiplication. For regeneration and fertility studies, the shoot-bearing calli on RN medium were transferred to Magenta boxes containing rooting medium. A dozen rooted plantlets were randomly chosen, and transferred to soil. These plants were first grown in humidified chambers, and then placed in the greenhouse to set seeds.

Preparation of subcultured callifor bombardment About 90 embryogenic calli, $1-2 \mathrm{~mm}$ in diameter, were placed at the center of a petri dish containing NB medium with $0.4 \mathrm{M}$ sorbitol (Vain et al., 1993). After incubation in this medium for 4 hours, the calli were immediately subjected to microprojectile bombardment using the particle accelerator Biolistic ${ }^{\text {बi }}$ PDS-1000/He (Bio-Rad laboratories, USA).

Microprojectile - mediated transformation The manufacturer's procedures for DNA coating and bombardment were followed with some modifications. Briefly, $25 \mu \mathrm{l}$ of freshly prepared $1.8-2.3 \mu \mathrm{m}$ gold particles $(60 \mathrm{mg} / \mathrm{ml}$; Alfa Research and Accessories. MA), $2.5 \mu 1$ of DNA $(1 \mu \mathrm{g} / \mu \mathrm{l})$, and $10 \mu \mathrm{l}$ of freshly prepared $0.1 \mathrm{M}$ spermidine were mixed in sequential order. While gently vortexing, $25 \mu \mathrm{l}$ of $2.5 \mathrm{M} \mathrm{CaCl}_{2}$ was slowly added. The mixture was incubated at room temperature for $10 \mathrm{~min}$. and pelleted at $12,000 \mathrm{rpm}$ in a microfuge for $1 \mathrm{~min}$. The supernatant was carefully removed, and the pellet was resuspended in $25 \mu \mathrm{l}$ of cold $100 \%$ ethanol. The suspension was gently vortexed, and an aliquot of $7.5 \mu \mathrm{l}$ of the suspension was drawn while vortexing and dispensed onto the center of a macrocarrier membrane. After drying briefly, the second 7.5- $\mu$ l aliquot was added to the same membrane. The membrane was allowed to dry completely in a desiccator for 2-5 min, and used immediately. After bombardment the calli were then separated from each other and incubated in the dark for $16-20 \mathrm{~h}$ at $25^{\circ} \mathrm{C}$. The bombarded calli were then transferred to $\mathrm{NB}$ medium containing $50 \mathrm{mg} / \mathrm{l}$ hygromycin (NBH50) and incubated in the dark at $25^{\circ} \mathrm{C}$ for 10-14 days. The small protuberances that grew from the calli were picked with aid of a dissecting microscope and transferred onto fresh NBH50 medium. After 14-day incubation, resistant calli were transferred to NBKNB medium containing $50 \mathrm{mg} / \mathrm{l}$ hygromycin, and incubated in the dark for 14-21 days. Finally, hygromycin resistant calli were regenerated on $\mathrm{RN}$ medium with $50 \mathrm{mg} / \mathrm{l}$ hygromycin under the conditions described above.

GUS assays GUS assays were carried out according to Jefferson (1987).

Southerm blot analyses Genomic DNA from rice leaves was extracted using a modified procedure of Dellaporta et al. (1983). Briefly, about $5 \mu \mathrm{g}$ of purified DNA was digested with the suitable restriction endonuclease in a volume of 100 $\mu l$ at $37^{\circ} \mathrm{C}$. The digested DNA was lyophilized to reduce the volume and subjected to electrophoresis in a 1\% agarose gel (Sambrook et al., 1989). After electrophoresis, the DNA was transferred to GeneScreen nylon membrane (NEN Research products, Boston, MA). Further fixation, prehybridization, hybridization and washing of the membrane were performed as per the manufacturer's recommendation. The ${ }^{32} \mathrm{P}$-labeled 
DNA probe for hybridization was prepared using Stratagene Prime-it ${ }^{\circledR}$ II random priming kit (Stratagene, La Jolla, California, USA) according to manufacture's instructions.

Segregation of the hygromycin transgene in RI seedlings About 30 seeds were dehusked and surface sterilized as described earlier, and germinated in Magenta boxes containing rooting medium under light in a chamber at $25^{\circ} \mathrm{C}$ for 1 week. When the piants were about $5 \mathrm{~cm}$ in height they were transferred to fresh rooting medium containing $50 \mathrm{mg} / \mathrm{l}$ hygromycin.

\section{Results}

Improved regenerability of selected TN1 subcultured calli.

The procedure for generating highly regenerable subcultured calli is described in Materials and Methods and schematically summarized in Figure 1 . The calli directly induced from mature embryos of TN1 (primary calli) are tightly attached to the surfaces of the embryos, and are highly heterogeneous in morphology (Fig 2A). Preliminary experiments showed that while the vast majority of the TN1 primary calli are recalcitrant to regeneration, a small number of plants can be consistently regenerated (Table 1). These initial studies suggested to us that there might be a small number of regenerable embryogenic calli among a given population of TN1 primary calli, and if so, it may be feasible to select and propagate such calli.

As shown in Figure 1, the dehusked and sterilized TN1 seeds were placed on NBKNB medium for 6 days to induce primary calli, followed by subculturing on the same fresh medium three times at 2-week intervals. At this point, at least five loosely attached globular calli, on average, emerged from the callus covered surface of each embryo (Figure $2 B$ ). We usually grow the calli to $2-3 \mathrm{~mm}$ in diameter. The subcultured calli were pooled, and then dispersed on fresh NBKNB plates marked with a grid. Regeneration tests with randomly sampled calli at this stage showed that $60-70 \%$ of the calli were able to regenerate and produce highly fertile plants (Table $1)$.

\begin{tabular}{ccc}
\hline $\begin{array}{l}\text { Number of } \\
\text { Subculture }\end{array}$ & $\begin{array}{c}\text { Regenerability } \\
(\%)^{\mathrm{a}}\end{array}$ & $\begin{array}{c}\text { Fertility } \\
(\%)^{\mathrm{b}}\end{array}$ \\
\hline 0 & 20 & 98 \\
4 & 64 & 93 \\
6 & 92 & 76 \\
7 & 96 & 62 \\
9 & 92 & ND \\
\hline
\end{tabular}

a number of plants per 100 calli

$b$ averaged percentage of fertile seeds per panicle

Table 1. Regenerability and fertility of TN1 subcultured calli.

To further improve the regeneration efficiency of the subcultured calli, we implemented a selection scheme to eliminate non-regenerable calli from the total population. One representative callus from each grid, which now contains a small number of sibling calli, was transferred to a corresponding grid on plates containing $\mathrm{RN}$ medium for regeneration. If a

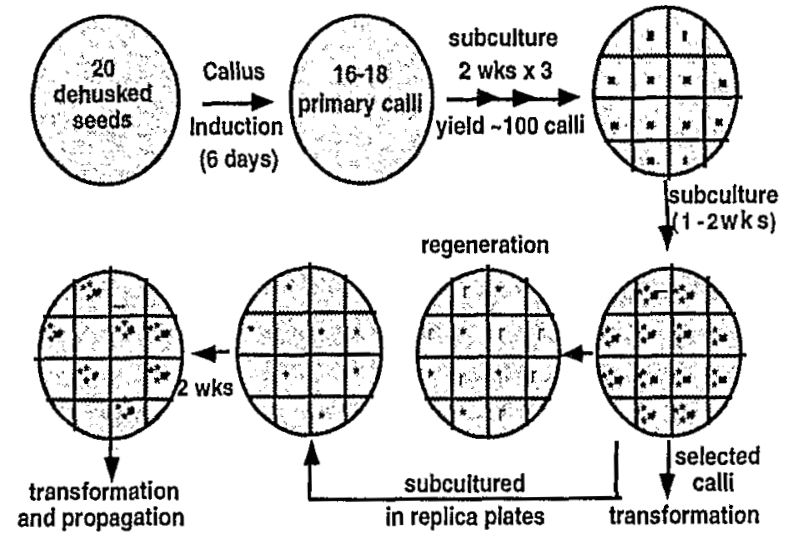

Figure 1: Schematic illustration of the procedure for generating embryogenic subcultured calli derived from TN1 mature seeds Twenty sterilized dehusked TN1 seeds were placed on NBKNB medium in a petri dish for 6 days to induce primary calli. About $80-90 \%$ of the seeds will produce primary calli on the scutellar surfaces. After removal of the primary calli from the embryos, they were further subcultured three times on the same media at two week intervals. At this stage, about 100 loosely attached globular calli (represented by "*") emerge. They are pooled and then individually placed on a gridded NBKNB plate for two more weeks. A small pool of morphologically uniform sibling calli emerge in each grid, and one callus from each pool is transferred to a corresponding gird on a plate containing RN medium for testing regenerability, If the representative callus regenerates to form shoots (indicated by " $r$ " on the RN plate), its sibling calli are saved for further multiplication and transformation.

representative callus regenerated small green shoots within 1-2 weeks, its sibling calli that remained on callus induction plates would be saved for further use. In this way, we generated a new pool of selected calli which exhibited high regenerability ( $>90 \%$ ). These calli maintained high levels of regenerability even after 5 additional rounds of subculture.

Morphology and fertility of plants regenerated from subcultured calli.

The subcultured calli that regenerated on $\mathrm{RN}$ plates were allowed to grow for about 3 weeks (Figure 2C). To examine the morphology and fertility of plants recovered from each batch of subcultured calli, a dozen such shoot-bearing calli were transferred to rooting medium and were further grown to maturity in the greenhouse. The plants were healthy (Figure 2D), and fertility among each batch of plants was uniform. The average percentage of fertile seeds per panicle for each group of plants was calculated, and as shown in Table 1 , the plants regenerated from the calli subcultured 6 times exhibited high levels of fertility.

\section{Transformation of subcultured TN1 calli}

The ultimate measurement of success of this work is whether the subcultured calli are suitable target materials for microprojectile mediated transformation. In order to assess the performance of the subcultured calli at the early phase of transformation, subcultured calli were bombarded with a mix of two plasmids, one carrying the $h p h$ gene and the other car- 
rying a uidA reporter gene. The efficiency of DNA uptake by the bombarded calli was examined by $\mathrm{X}$-gluc staining for transient expression of the uidA gene (Figure $2 \mathrm{E}$ ). We found that the DNA uptake by such calli was highly efficient. The number of blue spots per callus ranges from hundreds to nearly a thousand. Furthermore, the blue stains were distributed evenly on the bombarded surfaces of the calli. This might reflect the homogeneity of the micro callus-clusters proliferating on the surface of individual calli.

The first assessment of stable transformation efficiency was carried out by $\mathrm{X}$-gluc staining of the bombarded calli after growth on hygromycin-containing medium for 20 days. A significant percentage of the bombarded calli showed one or more blue cell clusters (Figure $2 F$ ). These results provided preliminary evidence that the subcultured calli can be stably transformed. Finally, some of the transgenic calli were capable of regenerating into R0 plants which exhibited GUS activity (Figure $2 \mathrm{G}$ ).

The efficiency of transformation of embryogenic subcultured calli was defined as the number of hygromycin-resistant RO plants regenerated from 100 calli bombarded under standardized conditions. The results obtained from three typical experiments are summarized in Table 2 . On average, three hygromycin-resistant R0 plants were obtained per 100 calli bombarded.

\begin{tabular}{cccc}
\hline $\begin{array}{c}\text { Experiment } \\
\text { number }\end{array}$ & $\begin{array}{c}\text { Calli } \\
\text { bombarded }\end{array}$ & R0 plants $^{\mathrm{a}}$ & $\begin{array}{c}\text { Transformation } \\
\text { efficiency }(\%)^{\mathrm{b}}\end{array}$ \\
\hline 1 & 273 & 3 & 1.1 \\
2 & 270 & 6 & 2.2 \\
3 & 270 & 15 & 5.6 \\
\hline
\end{tabular}

a. R0 plants resistant to hygromycin $(50 \mathrm{mg} / 1)$

b. Transformation efficiency is defined as number of hygromycin resistant R0 plants per 100 calli

Table 2. Transformation efficiency of embrogenic subcultures TN1 calli.

We routinely use $50 \mathrm{TN} 1$ seeds to initiate callus production. Within 10 weeks, thousands of highly embryogenic calli can be readily produced for microprojectile bombardment. By increasing the number of calli bombarded, we are able to routinely produce a large number of R0 plants for subsequent molecular and genetic analysis.

\section{Molecular analysis of R0 and R1 transgenic plants}

Genomic DNA samples of two independent R0 plants (R0-1 and R0-2) and two sibling R1 plants derived from R0-2 were analyzed for the presence of the hygromycin transgene (Figure 3). The southern blots contain DNA of each plant that was un-digested or digested with EcoRI or NcoI. Hybridization was carried out with a 32 p-labeled probe complimentary to the entire $h p h$ coding sequence. While the nontransformed TN1 genomic DNA showed no reaction, all lanes containing DNA samples from R0 and R1 plants showed positive hybridization signals. The $h p h$ gene probe specifically hybridized to the high molecular weight uncut DNA, indicating that the $h p h$ coding sequence is probably integrated into plant chromosomal DNA. Ncol digestion of the DNA from the two R0 plants showed two different digestion patterns, indicating the R0-1 and R0-2 are independent transformants. EcoR I digestion of the same DNAs further demonstrates that the intact $h p h$ coding sequence(s) are present in the genomes of the two RO plants.

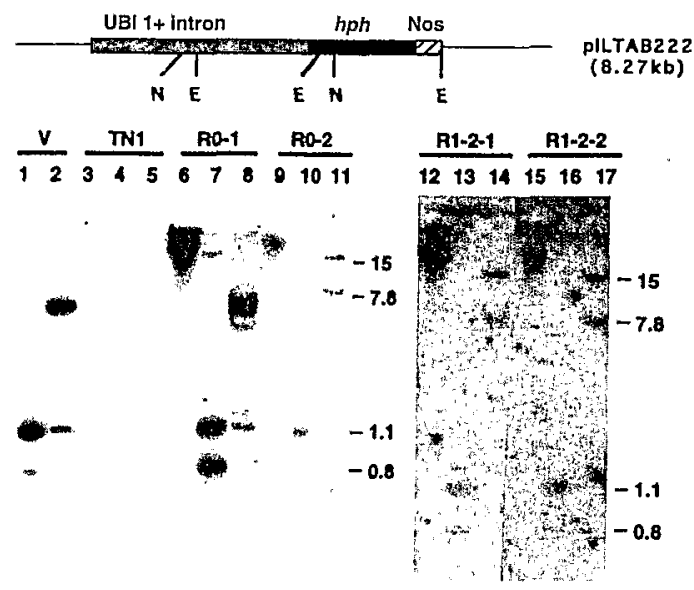

Figure 3: Genomic DNA blot analysis of R0 and R1 transgenic plants The restriction enzyme map of pILTAB222 is shown at the top. $\mathrm{E}$ and $\mathrm{N}$ denote EcoRI and NcoI sites, respectively. The DNA blot contains pILTAB222(V), the plasmid used for transformation, and the genomic DNA samples of a non-transgenic plant (TN1), two independent R0 lines (RO-1 and -2), and two R1 lines (R1-2-1 and $\mathrm{R} 1-2-2)$ derived from $\mathrm{R} 0-2$. The genomic DNA was visually quantitated and about $5 \mathrm{mg}$ was loaded in each lane. Lane 1 and 2: $100 \mathrm{pg}$ of pILTAB222 digested with EcoRI and NcoI, respectively. EcoRI digestion results in 1.1 and $0.8 \mathrm{~kb}$ bands, and $N$ col digestion yields a 6.9 and a $1.3 \mathrm{~kb}$ fragment. Lanes $3,6,9,12,15$ undigested genomic DNA of TN1, R0-1, R0-2, Rla, R1b, respectively. Lanes 4, 7, 10, 13, 16 are the same as their respective preceding lanes but cut with $E c o$ RI. Lanes $5,8,11,14,17$ are the same as lanes $3,6,9,12,15$ respectively, except digested with $N$ col. The numbers on the side of each panel indicate DNA size in $\mathrm{kb}$.

The R0-2 plant appears to have no more than two copies of the $h p h$ sequence, whereas the R0-1 plant contains multiple copies. The R1 plants derived from the $\mathrm{R} 0-2$ plants were analyzed for segregation of the $h p h$ resistance phenotype. The $R 1$ seedlings were grown on rooting medium containing 50 $\mathrm{mg} / \mathrm{l}$ hygromycin. After two weeks, two-thirds of the seedlings were resistant to hygromycin, and the rest were killed by the antibiotic (Figure 4). Genomic DNA samples extracted from two hygromycin-resistant RI seedlings were analyzed by southern blot hybridization, and DNA from both hybridized with the $h p h$ probe. The hybridization patterns appear to be identical to the parental R0-2 plant (Figure 3). In contrast, the DNAs of R1 seedlings that were subsequently shown to be sensitive to hygromycin showed no hybridization with the probe (data not shown). 


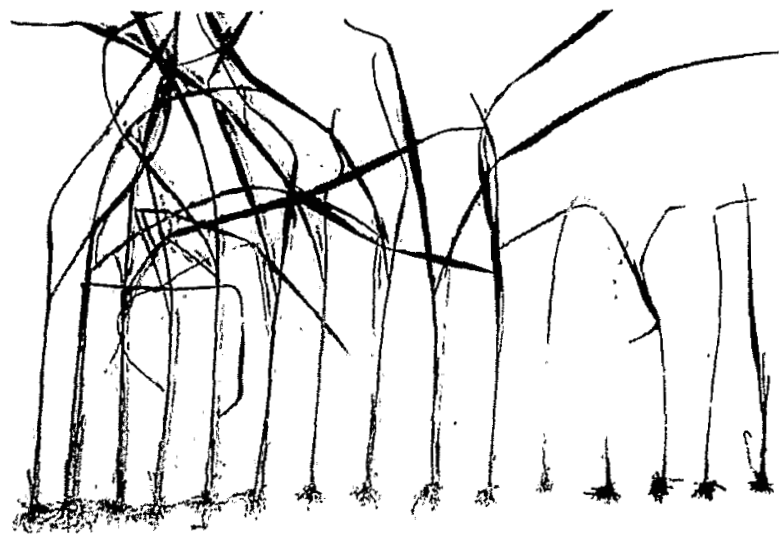

Figure 4: Hygromycin assay of the segregation of hph transgene in $R 1$ plants Fifteen $R 1$ seedlings derived from the $\mathrm{R} 0-2$ plant were transferred from half strength $M S$ medium to the same medium containing $50 \mathrm{mg} / 1$ hygromycin. After 2-week growth, 10 plantlets (1-10 from the right) showed normal growth while the other five were dead.

\section{Discussion}

The limiting factor of efficient transformation of indica rice is its recalcitrance to regeneration. A priori, a combination of two approaches can be used to overcome this problem. First, the regenerability of rice tissues used for transformation should be optimized. Second, the materials should be readily available in large quantity so that the odds of obtaining the desired number of transgenic plants can be improved. The method we described here is based on this rationale, and may provide useful guidelines for researchers attempting to transform other indica varieties. We routinely obtain 10 to 20 independent $R 0$ transgenic plants per 500 calli bombarded. More importantly, up to tens of thousands of embryogenic calli can be produced readily so that it is easy to scale up transformation experiments as necessary.

The transformation system described here minimizes the need for extensive tissue culture expertise, and can therefore be readily transferred to other laboratories. The selection process in this system is basically a self-taught process, which objectively distinguishes the regenerable calli from the nonregenerable ones. Once a researcher is familiarized with a particular rice variety, visual selection based on the morphology of the subcultured calli often is sufficiently reliable to select regenerable calli on a routine basis.

All the rice transformation techniques thus far developed involve some degree of callus induction and propagation following bombardment. While no single tissue culture protocol is suitable for callus growth and regeneration for all indica rice varieties, adjusting culture conditions will lead to optimized callus induction, propagation and plant regeneration. We recommend that the procedure reported here be used as a starting point for most indica varieties. For callus induction and propagation, one should vary the concentrations or types of cytokinins in the callus induction medium (Tian et al., 1994). In our experience with the TN1 variety, we found that the cells in the outer layers of subcultured calli proliferate well when cultured on NB medium but not on NBKNB medium, suggesting that the calli grown on NBKNB medium are derived from internal proliferating cells while the calli grown on the same medium without cytokinins (NB medium) are largely derived from the outer layers of cells. Likewise, regeneration conditions should also be adjusted. The calli usually regenerate better if they are grown on an optimized callus propagation medium containing proper concentration of certain type(s) of cytokinin before being transferred to regeneration medium.

We have examined the fertility of $\mathrm{R} 0$ transgenic plants derived from subcultured calli. About $50 \%$ of the R0 plant lines set seeds. The majority of those plants were 20 to $70 \%$ fertile. These fertility data are comparable to those observed in earlier transformation experiments using TN1 immature embryos (unpublished results). Furthermore, the majority of R1 seeds derived from RO plants have similar germination rates, and their seedlings are generally healthy.

\section{Acknowledgment}

We thank Drs. Alexandre de Kochko and Wenzhong Tian, for helpful discussion, and Ms Lili Chen for her excellent technical support. We also thank Dr. Peter Quail for pAHC17 and pAHC27 plasmids. This work was in part supported by a grant from the Rockefeller Foundation. C.F. was supported by ORSTOM, a French public research organization. E. S. was a recipient of the rice biotechnology postdoctoral fellowship from the Rockefeller Foundation.

\section{References}

Chan, M.-T., Chang, H.-H., Ho, S.-L., Tong, W.-F. and Yu, S.-M. (1993) Plant Mol. Biol. 22: 491-506.

Christensen, A. H., Sharrock, R. A. and Quail, P. H. (1992) Plant Mol. Biol. 18: 675-689.

Christou, P., Ford, T. L. and Kofron, M. (1991) Bio/Technology. 9: 957-962.

Chu C.C., Wang C.C., Sun, C.S., Hsu S.C., Yin, K.C., Chu, C.Y and Bi, F.Y. (1975) Sci. Sin. 18: 659-668.

Datta, S. K., Datta, K. and Potrykus, I. (1990) Plant Sci. 67: 83-88.

Gamborg O.L., Miller, R.A., and Ojima, K. (1968) J. Exp. Res. 50;151-158.

Ghosh Biswas, G. C., Burkhardt, P. K., Wünn, J., Klöti, A. and Potrykus, I. (1994) Plant Cell Reports 13(9): 528532.

Glaszmann, J. C. (1987) Theoretical and Applied Genetics. $74: 21-30$.

Hiei, Y., Ohta, S., Komari, T. and Kumashiro, T. (1994) The Plant Journal 6: 271-282

Jefferson, R. A. (1987) Plant Molecular Biology Reporter 5: 387.405 .

Li, L., Qu, R., Kochko de, A., Fauquet, C. and Beachy, R. N. (1993) Plant Cell Reports 12: 250-255.

Peng, J., Kononowicz, H. and Hodges, T. K. (1992) Theoretical and Applied Genetics. 83: 855-863.

Sambrook, J., Fritsch, E. F. and Maniatis, T. (1989) in Molecular cloning, a laboratory manual. Cold Spring Harbor Laboratory Press. pp 9.31-9.55.

Shimamoto, K., Terada, R., Izawa, T. and Fujimoto, H. (1989) Nature 338: 274-276.

Tian, W., Rancé, I. and Sivamani, E. (1994) Chinese Journal of Genetics 21(3): 1-9. 

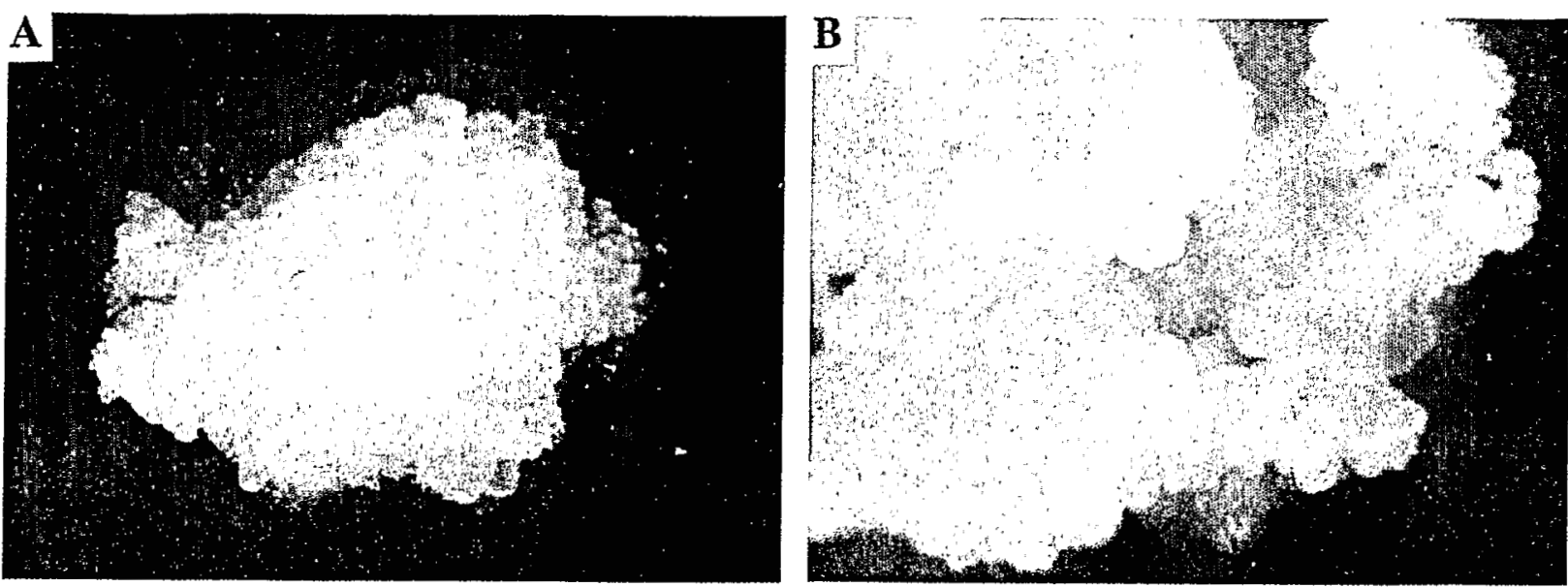

C
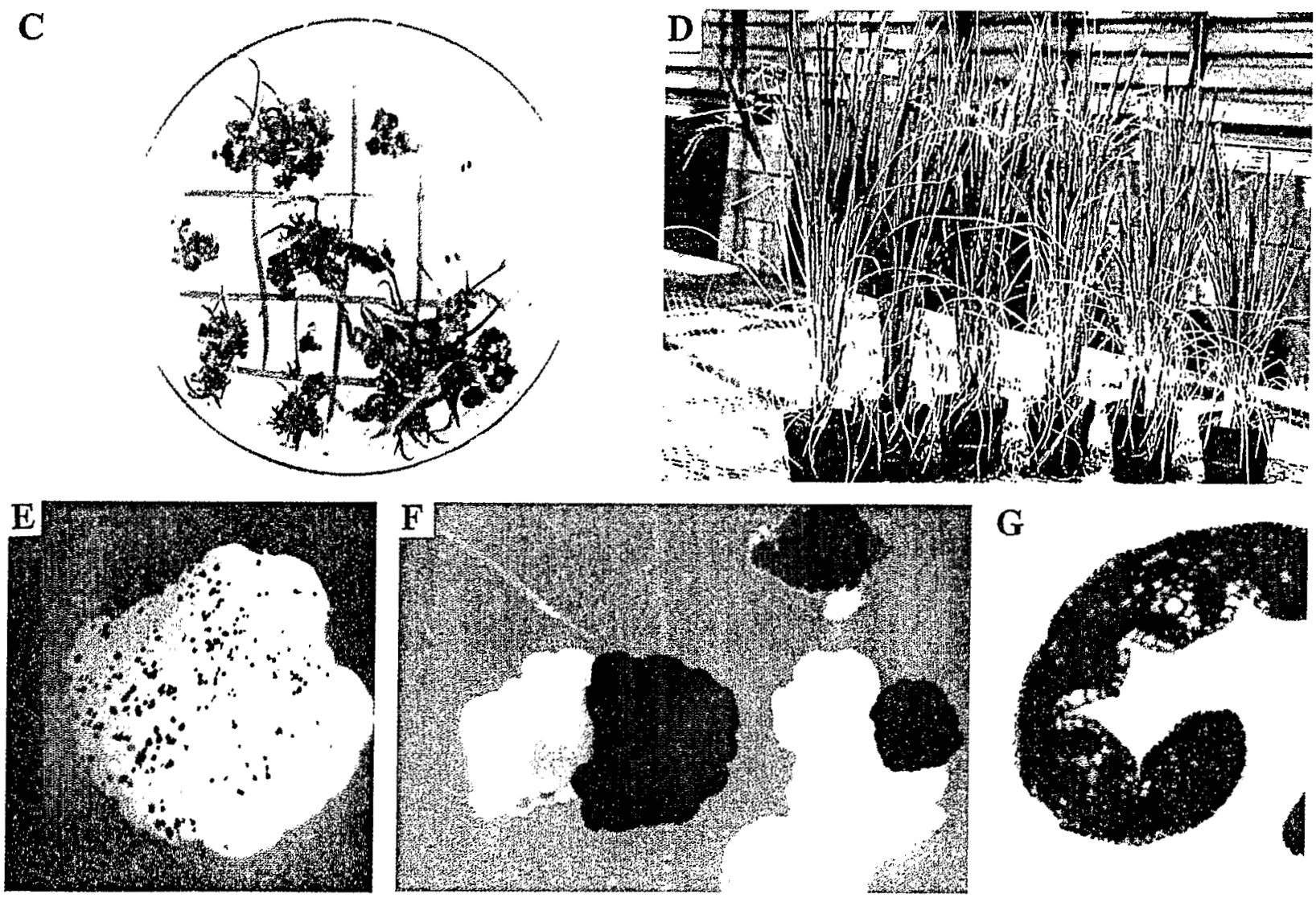

Toriyama, K., Arimoto, Y., Uchimiya, H. and Hinita, K. (1988) Bio/Technology. 6: 1072-1074.

Vain, P., NcMullen, M.D., Finer, J.J. (1993) Plant Cell Reports 12: 84-88.

Xu, X. and Li, B. (1994) Plant Cell Reports 13: 237-242

Zhang, H. M., Yang, H., Rech, E. L., Golds, T. J., Davis, A. S., Muliighan, B. J., Cocking, E. C. and Davey, M. R. (1988) Plant Cell Reports 7: 379-384.

Zhang, W. and Wu, R. (1988) Theor. Appl. Genet. 76: 835840.
Figure Legend

Figure 2: Evaluation of performance of subcultured TN1 calli in regeneration and transformation $2 \mathrm{~A}$ : the morphology of a primary callus 20 days after induction; $2 \mathrm{~B}$ : a subcultured callus 9 weeks after culturing on NBKNB medium; $2 \mathrm{C}$ : shoot formation of subcultured calli on RN plates to test for efficiency of regeneration; 2D: plants regenerated from subcultured calli; 2E: GUS staining of subcultured calli 48 hours after bombardment; $2 \mathrm{~F}$ : X-gluc staining of bombarded subcultured calli. The calli were grown for 20 days on NB medium containing $50 \mathrm{mg} / 1$ hygromycin; $2 \mathrm{G}$ : cross-section of a piece of transgenic rice leaf from an $\mathrm{R} 0$ plant. 


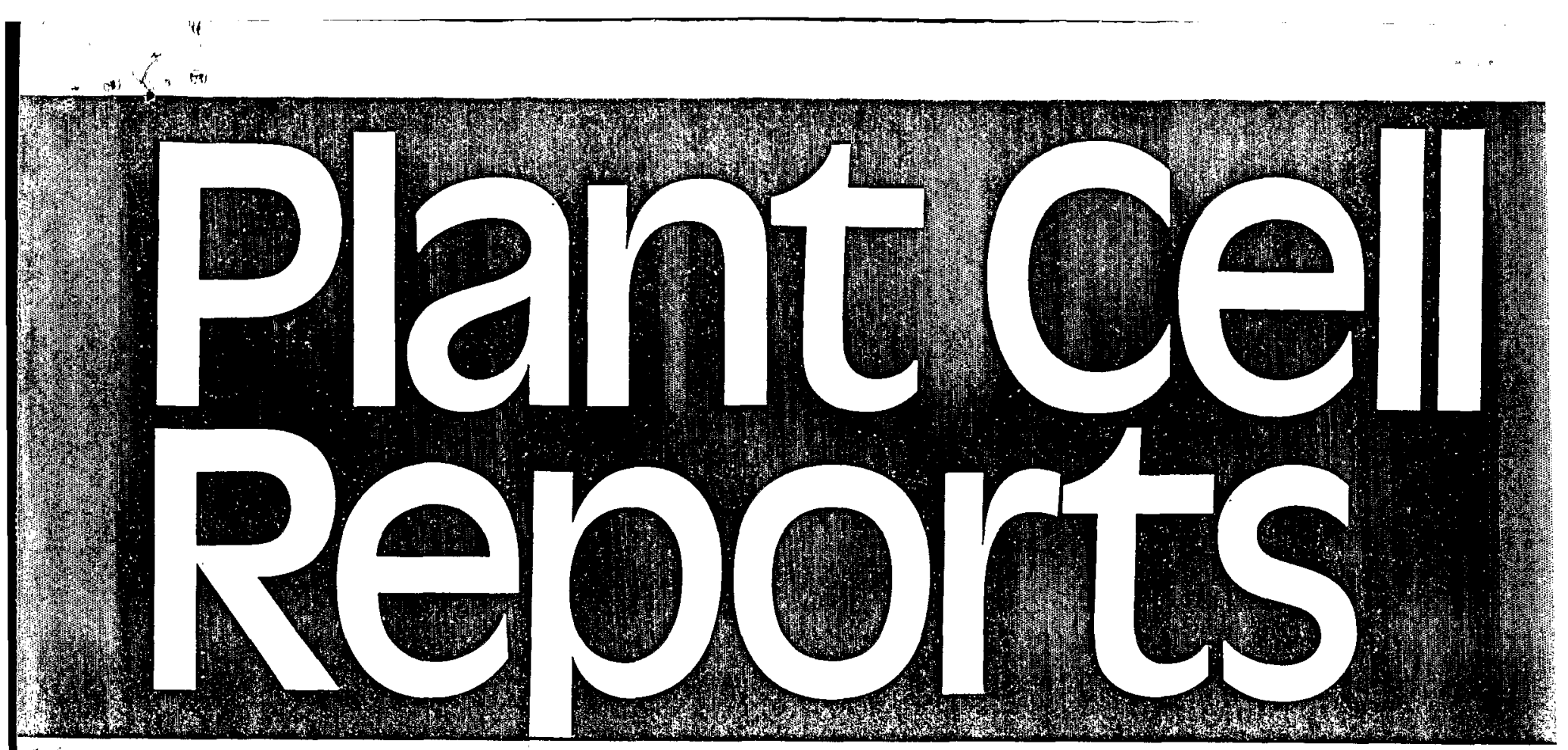

\section{Volume 15 Number 5,1996 \\ ISSN $0727-7714$ \\ MODAC = $D \triangle F R A$}

M. Chabaud, C. Larsonneau, C. Marmouget, T. Huguet: Transformation of barrel medic (Medicago truncatula Gaertn.) by Agrobacterium tumefaciens and regeneration via somatic embryogenesis of transgenic plants with the MtENOD12 nodulin promoter fused to the gus reporter gene

J. R. Kikkert, D. Hébert-Soulé, P. G. Wallace, M. J. Striem, B. I. Reisch: Transgenic plantlets of 'Chancellor' grapevine (Vitis sp.) from biolistic transformation of embryogenic cell suspensions

M. Yamazaki, L. Son, T. Hayashi, N. Morita, T. Asamizu, I. Murakoshi, K. Saito: Transgenic fertile Scoparia dulcis L., a folk medicinal plant, conferred with a herbicide-resistant trait using an Ri binary vector

E. Sivamani, P. Shen, N. Opalka, R. N. Beachy, C. M. Fauquet: Selection of large quantities of embryogenic calli from indica rice seeds for production of fertile transgenic plants using the biolistic method

A.S. Karthikeyan, K.S. Sarma, K. Veluthambi: Agrobacterium tumefaciens-mediated transformation of Vigna mungo (L.) Hepper

H. B. Jumin, N. Nito: Plant regeneration via somatic embryogenesis from protoplasts of six plant species related to Citrus

C. Orozco-Castillo, K.J. Chalmers, W. Powell, R. Waugh: RAPD and organelle specific PCR re-affirms taxonomic relationships within the genus Coffea

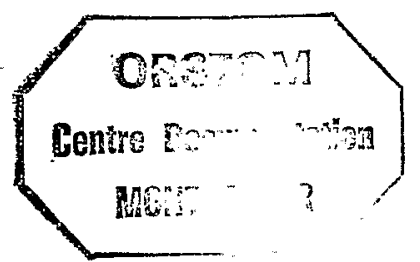

O. Erdelská, Z. Vidovencová, K. Erdelský: Cleavage polyembryos as explants for plant regeneration in wheat

M. Gutmann, J.P. Charpentier, P. Doumas, C. JayAllemand: Histological investigation of walnut cotyledon fragments for a better understanding of in vitro adventitious root initiation

S. N. R. Barr, L. A. Payne, M. F. B. Dale, M. J. Wilkinson: Predictive correlates of shoot regeneration from potato protoplast culture

M. M. Rao, G. L. Sita: Direct somatic embryogenesis from immature embryos of rosewood (Dalbergia latifolia Roxb.)

M. P. Chávez-Moctezuma, E. Lozoya-Gloria: Biosynthesis of the sesquiterpenic phytoalexin capsidiol in elicited root cultures of chili pepper (Capsicum annuum)

S. Nayak, B.K. Debata, S. Sahoo: Rapid propagation of lemongrass (Cymbopogon flexuosus (Nees) Wats.) through somatic embryogenesis in vitro

C.D. Rock, R.S. Quatrano: Lanthanide ions are agonists of transient gene expression in rice protoplasts and act in synergy with $A B A$ to increase $E m$ gene expression

C.E. Van Schaik, A. Posthuma, M.J. De Jeu, E. Jacobsen: Plant regeneration through somatic embryogenesis from callus induced on immature embryos of Alstroemeria spp. L.

Indexed in Current Contents/Agriculture, Biology and Environmental Sciences

PCRPD 815 (5) 305-380 (1996) January 1996 Printed on acid-free paper

\section{PH 133 \\ 12 FEV. 1996}


MANGA BASSIN VERSANT

165.

MARI MARE

166.

MAURITANIE

$77,80$.

MEDITERRANEE

40 .

MEXICO

112 .

MEXIQUE

$3,69,112$.

MONDE

$5,66,116,117$.

MONTPELLIER

2 .

MOPT I

58.

MOYEN ORIENT

108.

MOZAMB IQUE

103.

NAGBANGRE BARRAGE

162 .

NI AMEY

177.

\section{NIAMEY REGION}

$60,123$.

NIARI VALLEE DU

68.

NICARAGUA

143.

NIGER

$60,62,77,79,123,137,144,166,177$.

NIGER BOUCLE

79 .

NIGERIA

$79,81,85,86,87$.

NIL VALLEE

93,96 .

OCEAN INDIEN ILES

$68, I 06$. 